\title{
Short- and Long-Range Correlations in Beat Rate Variability of Human Pluripotent-Stem-Cell-Derived Cardiomyocytes
}

\author{
Jiyeong Kim ${ }^{1}$, Jukka Kuusela ${ }^{2}$, Katriina Aalto-Setälä ${ }^{2}$ Esa Räsänen ${ }^{1}$ \\ ${ }^{1}$ Tampere University of Technology, Tampere, Finland \\ ${ }^{2}$ BioMediTech, University of Tampere, Tampere, Finland
}

\begin{abstract}
A healthy heart exhibits fractal, i.e., long-range correlated fluctuations in heart rate variability (HRV). It is recently shown that fractal dynamics is also an intrinsic feature of human-induced pluripotent stem cell-derived cardiomyocytes (hiPSC-CMs). In this study, we investigate short- and long-range correlations in beat rate variability (BRV) of hiPSC-CMs, obtained from a healthy subject and symptomatic and asymptomatic long $Q T$ syndrome patients. It is shown that it is important to distinguish correlation properties in short and long time scales, as the scaling exponents are significantly different and also behave differently in the acute exposure to pharmacological compounds that modulate $\beta 1$-adrenoreceptors and cardiac ion channel generating delayed, outwardly rectifying $\mathrm{K}^{+}$current $\left(I_{K s}\right)$. While long-range scaling is sensitive to the drug exposure, short-range scaling is barely affected.
\end{abstract}

\section{Introduction}

Self-similar patterns appear in many physiological time series, such as R-R intervals, characterized by a powerdecaying relation with a non-integer scaling exponent. Such properties are referred loosely as fractals, or having long-range correlation.

In physiological systems, nonlinear complexity is often related to the system's autonomic regulation and robustness against external perturbation. Many studies in the past have confirmed the presence of fractal scaling in heart rate variability (HRV) and its alteration due to aging [1] and cardiac disorders, e.g., [2]. It was shown that isolated heart cells, lacking any neural inputs, also exhibit powerlaw scaling, suggesting that fractal-like beat rate fluctuation is an intrinsic feature of heart cells [3].

Dynamics of spontaneously beating human heart cells, or cardiomyocytes (CMs), has not been widely explored until recently, when in vitro measurements of isolated human heart cells were made possible with newly developed stem cell technologies. According to the revolutionary scheme that led to the Nobel Prize in Medicine in 2012, human-induced pluripotent stem cells (hiPSCs) can be obtained from adult tissues such as skin and give rise to any cell type in the body, including CMs [4].

In our previous work [5], we measured and analyzed the beat-to-beat interval series of hiPSC-derived CMs (hiPSC$\mathrm{CMs}$ ) and R-R interval of electrocardiogram (ECG), obtained from a healthy individual and symptomatic and asymptomatic long QT syndrome (LQTS, type 1) patients. LQTS is a cardiac disease, characterized by prolonged QT intervals in ECG. The inherited forms of LQTS are a result of mutations in the cardiac ion channel coding genes [5]. We also tested the effect of pharmacological compounds, applied directly to the cultured hiPSC-CMs. In this study, we extend the work further to consider short- and longrange correlation in different scale regimes, rather than computing one global scaling exponent over all scales, and assess the crossover behavior.

\section{Data and Methods}

\section{1. hiPSC-CM preparation}

The hiPSCs are generated as described in [6]. The LQT1-specific hiPSCs are derived from LQTS patients' skin fibroblasts carrying G589D missense mutation in KCNQ1 $[7,8]$. The skin biopsies are obtained from a 55-year-old female healthy individual, a symptomatic 41year-old female LQTS patient, and an asymptomatic 28year-old LQT-mutation carrier. Both symptomatic and asymptomatic LQTS patients are on bisoprolol medication. The hiPSCs are cultured and differentiated into CMs as described in [8]. In this study, 30-40 days old hiPSCCMs are used. The study is approved by the ethical committee of Pirkanmaa Hospital District (R08070).

\section{2. hiPSC-CM and ECG data acquisition}

Spontaneously beating hiPSC-CM clusters are measured with 6-well multielectrode array (MEA), as described in [5]. After 30 minutes of baseline measurements, 
Bisoprolol ( $\beta$-blocker), ML277 ( $\mathrm{I}_{\mathrm{Ks}}$ activator), and JNJ303 $\left(\mathrm{I}_{\mathrm{Ks}}\right.$ blocker) are applied. They are dissolved in either dimethyl sulfoxide (DMSO) or $\mathrm{H}_{2} \mathrm{O}$. Five minutes after each addition of a drug, field potential data is recorded for 30 minutes. The data is then analyzed by Cardiomyocyte MEA Data Analysis (CardioMDA) [9] to produce the hiPSC-CM cluster's beat-to-beat interval series. Typical length of the hiPSC-CM data is about 1000-3000 beats. ECGs of the individuals, whose hiPSC-CMs are studied, are recorded using MARS-Holter. The ECG recordings used here contain about 100000 beats.

\subsection{Detrended fluctuation analysis}

We apply detrended fluctuation analysis (DFA), originally developed in Ref. [10]. DFA is a reliable method to quantify long-range correlation in a time series that may be non-stationary.

In DFA, integrated time series (profile) is divided into non-overlapping segments, from which a polynomial trend is estimated by least-square fitting and subtracted, removing any monotonous trend. The fluctuation function is defined by the root-mean-square of the variance of the residuals of the profile. Since $F(s) \sim s^{\alpha}$ in the presence of power-law scaling, plotting $F(s)$ against $s$ in log-log scale and calculating the slope of the linear fit yields the scaling exponent $\alpha$. The algorithm used here is described more thoroughly in Ref. [11].

The scaling exponent $\alpha$ describes the nature of the correlation present in the data. The white noise with no correlation and Brownian noise are characterized by $\alpha=0.5$ and $\alpha=1.5$, respectively. $0.5<\alpha<1.5$ indicates long-range correlation, i.e., fractal correlation, while $\alpha<0.5$ corresponds to long-range anti-correlation [11]. It is important to note here that time series may require more than one scaling exponent to describe different behaviors at different time scales. This crossover can be detected as change in slope in the log-log plot of $F(s)$ against $s$.

In order to determine the statistical significance in the changes caused by the acute application of the drugs and differences between the cell groups and short- and longrange scaling exponents, paired sample t-test and independent (unpaired) t-test are used, respectively. The levels of significance are represented as asterisks: $(*) \mathrm{p}<0.05,(* *)$ $\mathrm{p}<0.01$, and $(* * *) \mathrm{p}<0.001$.

\section{Results and Discussion}

\subsection{Beat rate variability of hiPSC-CMs}

Beat rate variability (BRV) of hiPSC-CMs , derived from three individuals with different health conditions are examined: healthy control, also called wild type
(WT), symptomatic LQTS patient, and asymptomatic LQT-mutation carrier.

Applying DFA, we compute the scaling exponents $\alpha_{1}$ for time scales less than 16 beats, and $\alpha_{2}$ for scale longer than 40 beats. They represent short-range and longrange correlation respectively. In the extant literature on crossover phenomena (e.g., [2]), $\alpha_{1}$ is usually defined by scales less than 10 beats, but we choose 16 beats as our threshold because crossover points occur after 16 beats in most cases. DFA exponents are averaged over 38 data sets of WT-CMs, 34 sets of symptomatic LQT-CMs, and 58 sets of asymptomatic LQT-CMs. The results are summarized in Table 1.

Scaling behavior of the healthy control, also called wild type (WT), and asymptomatic LQT-CMs are almost the same. On the other hand, symptomatic LQT-CMs has significantly smaller $\alpha_{1}(\mathrm{p}<0.01$, compared to asymptomatic LQT-CMs), while the $\alpha_{2}$ is comparable to those of WTand asymptomatic LQT-CMs. The difference between mean $\alpha_{1}$ and mean $\alpha_{2}$ is statistically significant in all cell groups $(\mathrm{p}<0.001)$. The significant change in the scaling behavior at short and long time scales indicates the existence of crossover. Thus, it is appropriate to describe the BRV of the hiPSC-CMs with two and possibly more scaling exponents.

Table 1. Average of DFA $\alpha_{1}$ and $\alpha_{2}$ values describing short- and long-range correlation in BRV of hiPSC WTand LQT-CMs.

\begin{tabular}{lcc}
\hline Cell group & mean $\alpha_{1}$ & mean $\alpha_{2}$ \\
\hline \hline Wild type (healthy) & $0.93 \pm 0.19$ & $1.10 \pm 0.15$ \\
LQT (symptomatic) & $0.84 \pm 0.15$ & $1.05 \pm 0.23$ \\
LQT (asymptomatic) & $0.94 \pm 0.17$ & $1.06 \pm 0.20$ \\
\hline
\end{tabular}

Table 2. DFA $\alpha_{1}$ and $\alpha_{2}$ and their $95 \%$ confidence intervals, calculated from R-R intervals of ECG, recorded from the healthy control, symptomatic LQTS patient, and asymptomatic LQT-mutation carrier.

\begin{tabular}{lcc}
\hline ECG group & $\alpha_{1}$ & $\alpha_{2}$ \\
\hline \hline Control (healthy) & $1.12 \pm 0.03$ & $1.33 \pm 0.03$ \\
LQT (symptomatic) & $1.21 \pm 0.01$ & $1.08 \pm 0.02$ \\
LQT (asymptomatic) & $0.90 \pm 0.01$ & $1.00 \pm 0.02$ \\
\hline
\end{tabular}

\subsection{Comparison with ECG}

R-R intervals, extracted from ECGs, are used to study heart rate variability (HRV). Corresponding DFA results are shown in Table 2 and illustrated in Fig. 1, presenting short-range and long-range $\alpha$ s and their $95 \%$ confidence intervals. The scaling properties are comparable between 

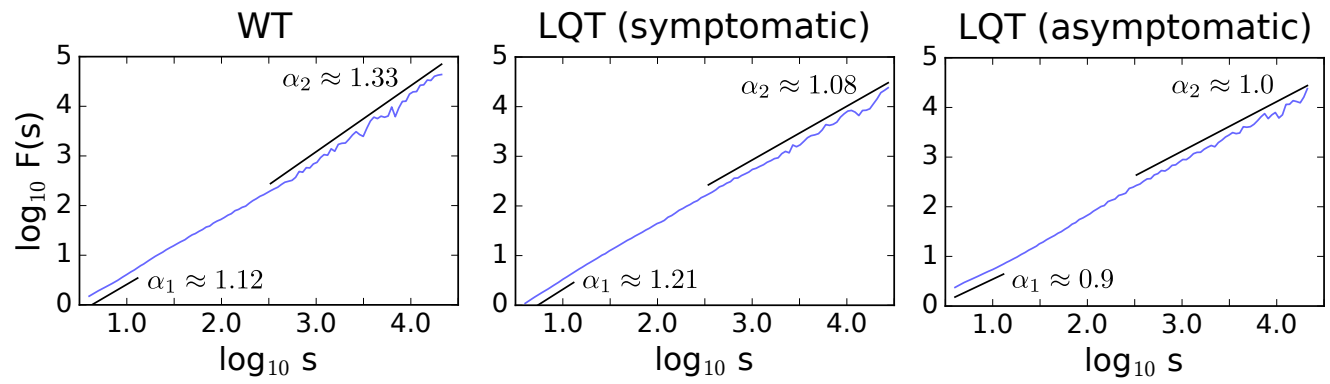

Figure 1. Fluctuation as a function of scale in $\log 10$ scales, computed by DFA algorithm, for the healthy control (WT) and LQTS patients. $\alpha_{1}$ and $\alpha_{2}$ are calculated from least-square fitting on small scale ( $<14$ beats) and large scale ( $>300$ beats).

cellular and heart levels, especially for asymptomatic LQTCMs. Healthy control has higher $\alpha_{1}$ and $\alpha_{2}$ in ECG than at the cellular level. The significantly reduced $\alpha_{1}$ of symptomatic LQT-CMs is not observed in ECG data.

The findings confirm that hiPSC-CMs exhibit intrinsic BRV with fractal scaling, and there are other internal and external inputs affecting the fractal dynamics beyond the cellular level: at the heart's in situ level, sympathetic and parasympathetic neural inputs regulate the heart rate, thus affecting the fractal behavior.

\subsection{Effects of pharmacological compounds}

Three different pharmacological compounds are applied to the hiPSC-CMs. Results are summarized in Fig. 2.

$\boldsymbol{\beta}$-blocker Bisoprolol is a $\beta 1$-adrenoreceptor selective $\beta$-blocker that is the standard treatment of choice for LQTS patients. Acute application of bisoprolol leads to a statistically significant increase in the long-range correlation in all the cell groups. Above the therapeutic concentration $(260 \mathrm{nM})$, the effect attenuates or remains at the same level for WT- and asymptomatic LQT-CMs. $\alpha_{2}$ increases further towards Brownian noise $(\alpha=1.5)$ for symptomatic LQT-CMs, but not significantly.

$\mathbf{I}_{\mathrm{Ks}}$ activator ML277 is an ion-channel activator, known to shorten the action potential duration of CMs. The application of ML277 to the hiPSC-CMs leads to a significant increase in the long-range correlation in WTand asymptomatic LQT-CMs. In all the cell groups, higher concentration does not increase $\alpha_{2}$ any further. There is no significant change in short-range correlation.

$\mathbf{I}_{\mathrm{Ks}}$ blocker $\mathbf{J N J 3 0 3}$ is an effective and specific $\mathrm{I}_{\mathrm{Ks}}$ blocker. Though JNJ303 has the opposite effect on $\mathrm{I}_{\mathrm{Ks}}$ channel, its effect on the fractal scaling is similar to that of ML277. Similar to other compound, application of JNJ303 leads to significant increase in $\alpha_{2}$. The effect attenuates slightly or remains the same at higher concentration. Again, no significant changes are observed in short-range correlation.

WT- and asymptomatic LQT-CMs seem to be more sen- sitive than symptomatic LQT-CMs to the drug exposures. In summary, acute application of pharmacological compounds that modulate $\beta 1$-adrenoreceptors and cardiac ion channel generating $\mathrm{I}_{\mathrm{Ks}}$ alter the CM cluster's fractal complexity by increasing long-range correlation, but independently of the applied dose. There is, however, no significant alteration in the short-range scaling due to the compounds.

We have also performed vehicle control experiments, in which CM clusters are measured without any drug exposure, but with vehicle solutions, DMSO or $\mathrm{H}_{2} \mathrm{O}$. We observe no statistically significant changes in $\alpha_{1}$ and $\alpha_{2}$, when vehicle solutions are added every 30 minutes. Therefore, significant changes in the scaling properties caused by drug exposures reflect the intrinsic effects of the pharmacological compounds themselves.

\section{Conclusion}

We have confirmed that fractal scaling properties are indeed intrinsic at the cellular level, also for LQT-CMs. In particular, asymptomatic LQT-CMs show scaling behavior very similar to that of healthy CMs, suggesting that the intrinsic mechanism contributing to fractality is not altered by the presence of LQT-specific genetic mutation.

In this study, we have had a particular focus on two scaling exponents to describe BRVs at short and long time scales. The significant difference in the short and long range $\alpha$ s indicates that it is appropriate to use two (or even more) scaling exponents. Our results show that the effects of drug exposure are different for the scaling properties at short and long range: while short-range scaling is unaltered by the modulation of $\beta 1$-adrenoreceptors and cardiac ion channels generating $\mathrm{I}_{\mathrm{Ks}}$, the long-range scaling is sensitive to the drug exposure. Generally, the long-range scaling exponents increase towards Brownian noise, independently of the drug concentration. 


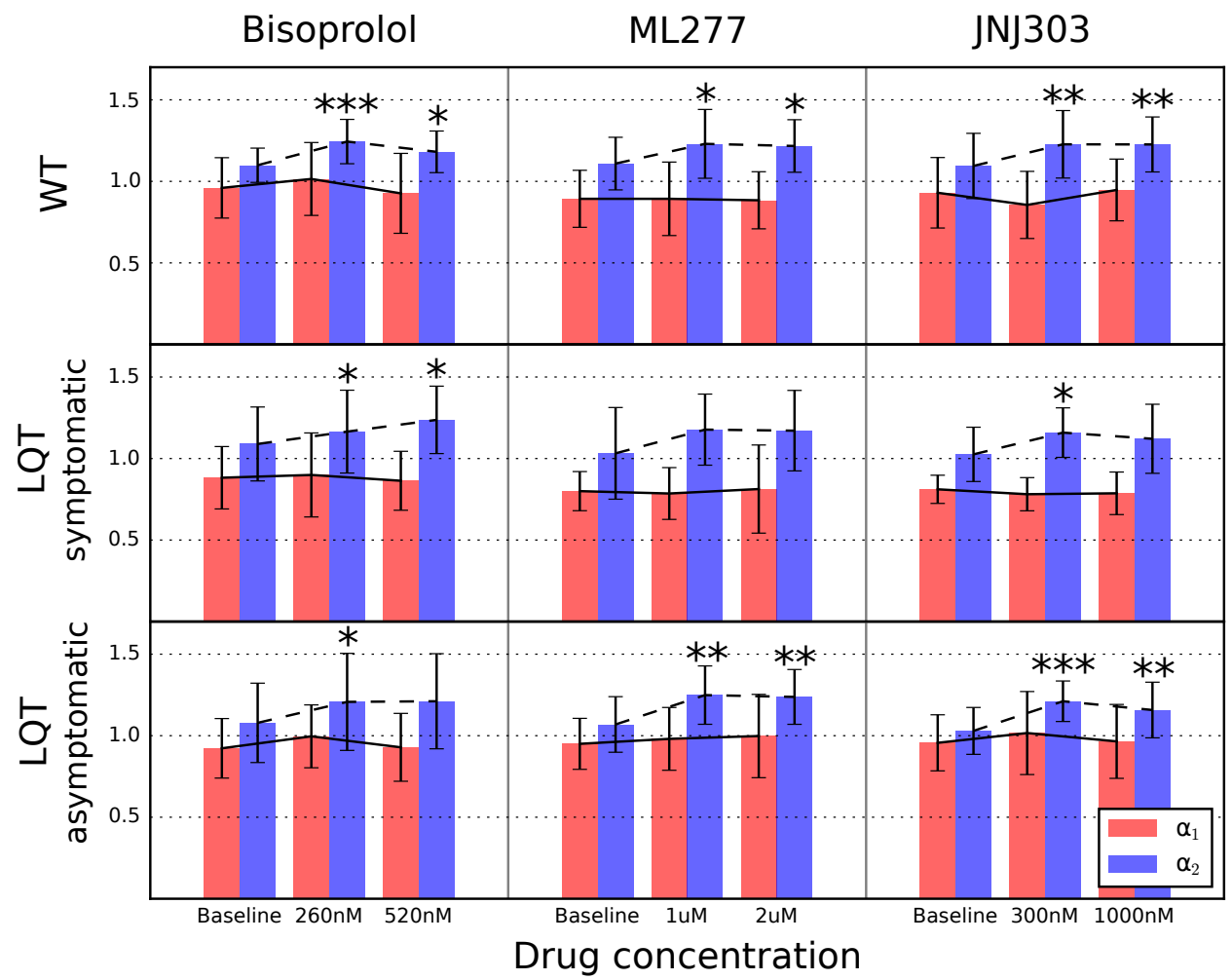

Figure 2. Change in average short-range correlation $\alpha_{1}$ and long-range correlation $\alpha_{2}$ as a function of drug concentration, by cell groups and compounds. Significance levels are indicated by $\left(^{*}\right) p<0.05,(* *) p<0.01,\left({ }^{* *}\right) p<0.001$.

\section{References}

[1] Otsuka K, Cornelissen G, Halberg F. Age, gender and fractal scaling in heart rate variability. Clinical Science 1997; 93(4):299-308.

[2] Peng CK, Havlin S, Stanley HE, Goldberger AL. Quantification of scaling exponents and crossover phenomena in nonstationary heartbeat time series. Chaos An Interdisciplinary Journal of Nonlinear Science 1995;5(1):82-87.

[3] Mandel Y, Weissman A, Schick R, Barad L, Novak A, Meiry G, Goldberg S, Lorber A, Rosen MR, ItskovitzEldor J, et al. Human embryonic and induced pluripotent stem cells-derived cardiomyocytes exhibit beat rate variability and power-law behavior. Circulation 2012; CIRCULATIONAHA-111.

[4] Yamanaka S. Induced pluripotent stem cells: past, present, and future. Cell stem cell 2012;10(6):678-684.

[5] Kuusela J, Kim J, Räsänen E, Aalto-Setälä K. The effects of pharmacological compounds on beat rate variations in human long qt-syndrome cardiomyocytes. Stem Cell Reviews and Reports 2016;12(6):698-707.

[6] Takahashi K, Tanabe K, Ohnuki M, Narita M, Ichisaka T, Tomoda K, Yamanaka S. Induction of pluripotent stem cells from adult human fibroblasts by defined factors. cell 2007; 131(5):861-872.

[7] Kiviaho AL, Ahola A, Larsson K, Penttinen K, Swan H, Pekkanen-Mattila M, Venäläinen H, Paavola K, Hyttinen
J, Aalto-Setälä K. Distinct electrophysiological and mechanical beating phenotypes of long qt syndrome type 1specific cardiomyocytes carrying different mutations. IJC Heart Vasculature 2015;8:19-31.

[8] Kuusela J, Kujala VJ, Kiviaho A, Ojala M, Swan H, Kontula K, Aalto-Setälä K. Effects of cardioactive drugs on human induced pluripotent stem cell derived long qt syndrome cardiomyocytes. SpringerPlus 2016;5(1):234.

[9] Pradhapan P, Kuusela J, Viik J, Aalto-Setälä K, Hyttinen J. Cardiomyocyte mea data analysis (cardiomda)-a novel field potential data analysis software for pluripotent stem cell derived cardiomyocytes. PloS one 2013;8(9):e73637.

[10] Peng CK, Buldyrev SV, Havlin S, Simons M, Stanley HE, Goldberger AL. Mosaic organization of dna nucleotides. Physical review e 1994;49(2):1685.

[11] Kantelhardt JW, Koscielny-Bunde E, Rego HH, Havlin S, Bunde A. Detecting long-range correlations with detrended fluctuation analysis. Physica A Statistical Mechanics and its Applications 2001;295(3):441-454.

Address for correspondence:

Jiyeong Kim

Laboratory of Physics, Tampere University of Technology

Korkeakoulunkatu 10, 33720 Tampere

jiyeong.kim@tut.fi 